\title{
A Vibration Signal Processing of Large-scale Structural Systems Based on Wireless Sensor
}

\author{
https://doi.org/10.3991/ijoe.v13i05.7050 \\ Lian Xue \\ WuHan Technology And Business University, Wuhan, China \\ vph9287506@126.com \\ Cheng-song $\mathrm{Hu}$ \\ WuHan Technology And Business University, Wuhan, China \\ vfard9538364@126.com
}

\begin{abstract}
The inherent characteristics of large-scale structural system are also called modal parameters, which include natural frequency, damping ratio and vibration mode. They are the basis for analyzing dynamic characteristics of large-scale structural system. Modal Parameter Identification is a modern method, and it is used to identify the vibration signals. At present, the problem of large-scale structural system security is paid more and more attention to, so the method of modal parameter recognition is very significant. A fast integral method is put forward to eliminate the trend item of vibration signals, and the vibration signals are collected through the wireless sensor network (acceleration signal), so as to obtain the integrated vibration signal (speed and displacement signal). The polynomial fitting method is applied to eliminate the trend items in the sampling integral, and improve the operation speed and accuracy by the relationship among the various coefficients. Then, they are discretized to meet the wireless sensor network requirements of "online" processing and analysis. Through the simulation of acceleration signals based on finite element modeling and the processing of actual acquisition acceleration signals based on wireless sensor network, the effectiveness of this method was verified. As a result, the precision effect by sampling frequency and the data length is summarized.
\end{abstract}

Keywords-wireless sensor, large-scale structure, modal parameter identification

\section{Introduction}

Acceleration signals, velocity signals and displacement signals all are very significant vibration signals [1]. The dynamic performance (natural frequency, damping ratio, modal mode, etc.) of system can be obtained by analyzing the vibration signal [2]. Through the displacement signals, we can not only check the structural stiffness (whether the actual displacement exceeds the allowable displacement limit), but also can estimate the load ability of system (the ratio of measured value and assay value). Moreover, acceleration signal is easily obtained in the acquisition process, but dis- 
placement signal acquisition often has many problems, like expensive high precision equipment, sensor installation, difficult on small vibration displacement acquisition and other issues. Therefore, we can use acceleration signal of relative easy acquisition, to obtain the signal of relative difficult acquisition based on the integral relation between acceleration, velocity and displacement [3]. Although the integral relation between them is very clear, in the actual collection process, the sensor will produce zero drift and zero drift is not fixed, which make this problem more complex [4]. Obtaining the more "real" displacement signal through the actual acceleration signal has become the research direction of many scholars.

With the rise of wireless sensor network, the acceleration signal is easily gathered in the condition of high frequency sampling. Through collected acceleration signal to obtain the velocity displacement signal is still an important part in vibration signal processing. The main method is based on frequency domain method and time domain method. How to eliminate the trend item in a large extent is still the main problem. But frequency domain method is easy to cause the truncation error, such as leakage. This phenomenon will occupy more resources because wireless sensor networks need us to analyze and tackle the problem online. Therefore, this paper focuses on the analysis of the causes of errors and how to better eliminate the trend item.

\section{State of the art}

With the rapid development of computers, people slowly began to analyze such problems in the time domain. In 1996, Faulkner et al. provided evidence that sampling frequency was a key factor in dynamic testing. It can calculate the displacement signal by the integral relationship between acceleration and velocity and displacement. In 2007, AdrewSmyth and Meiliang Wu proposed that the acceleration signal contained low frequency noise. In addition, the displacement signal contains high frequency noise. Based on the multi-proportional Kalman filtering method, it obtains the displacement signal by the acceleration signal. In 2009, Liu Desheng put forward a new idea based on wireless sensor network to achieve sound signal extraction and transmission, and it realized the transformation from independence to system. In 2015, Yang Tong proposed a new photoelectric signal acquisition system. The system takes STM32F103ZET6 as the master, and the nRF24L01 is a wireless transmission module. It gives the system hardware and software implementation method, and applied it to the silicon photovoltaic cell (PC50-6) as a sensor component of the signal acquisition. The results show that the new system has improved the speed and precision of signal acquisition, and it can meet the requirements of the system. In 2016, Wang Wei et al. proposed a sensor network singular signal detection algorithm combining wavelet analysis and chaos theory. It uses wavelet analysis to decompose the sensor network singular signals. The signal components are decomposed by chaos theory. The data mining technology is used to detect the singularity of the sensor network. The results show that the singularity of the sensor network is improved obviously. It reduces the singular signal false detection rate and missed rate, thus ensuring the wireless sensor network communication security. 
In summary, it is an important part of the vibration signal processing to obtain the velocity and displacement signals by collecting the acceleration signals. Although there is only a small amount of literature on how to eliminate the trend items, the question of how to improve the speed and accuracy of the operation, how to determine the initial value of velocity and displacement, and how to solve the zero dynamic drift is still not solved. Therefore, A fast integral method is put forward to eliminate the trend item of vibration signals, and the vibration signals are collected through the wireless sensor network (acceleration signal), so as to obtain the integrated vibration signal (speed and displacement signal). The polynomial fitting method is applied to eliminate the trend items in the sampling integral, and improve the operation speed and accuracy by the relationship among the various coefficients. Then, they are discretized to meet the wireless sensor network requirements of "online" processing and analysis. Through the simulation of acceleration signals based on finite element modeling and the processing of actual acquisition acceleration signals based on wireless sensor network, the effectiveness of this method was verified. As a result, the precision effect by sampling frequency and the data length is summarized.

\section{Modal parameter identification}

\subsection{Vibration displacement signal}

Vibration is a common natural phenomenon, and it is the reciprocating motion of objects or structures over time with respect to their equilibrium position. Although the vibration is beneficial, it will influence equipment performance, equipment life and the performance of building and large structure.

Vibration signal processing is to analyze and process the collected data in various ways in order to obtain the required information we need, so as to solve or prevent the related problems caused by vibration based on effective measures.

For nearly 30 years, vibration signal processing has undergone the process of the artificial analysis of analog signal and digital signal processing. The analysis of the vibration signal has developed rapidly on the basis of theory and technology.

With the appearance of structural health monitoring and the development of wireless sensor networks, more and more data can be obtained from the observed objects in real time, so as to understand the health of the observed objects. However, in many cases, acquisition of certain data is very difficult, even unable be directly measured, thereby other data processing methods should be considered.

Vibration displacement is one of the significant indicators to reflect the structural health. At present the common methods are precision level method, hanging hammer method, static level method, integrated grating interference micro-displacement measurement method [5-6]. But in many cases the measurement of vibration displacement is very difficult and cannot be measured directly. For example, it is almost impossible to measure the vibration displacement of roof to ground. In addition, it is also very difficult to obtain the vibration displacement of bridge and monitor the health of bridge when the bridge is using. Even if the instrument can be measured by 
precision instruments, the cost is still too high. It is an effective method to obtain the vibration displacement by acceleration signal [7-8]. However, the speed signal and the displacement signal are obtained by the acceleration signal. The acquired acceleration signal is converted to the frequency domain by fast Fourier transform. We integrate the converted acceleration signal in frequency domain based on calculus's properties, so as to obtain speed and displacement signals which belong to frequency domain, then convert it to time domain by Inverse Fourier Transform. The direct current signal of data collection is also can be converted by the signals in frequency domain. The processing of the signal undergoes positive transform and inverse transformation of the transform domain, thereby causing error.

Due to the wide application of wireless sensor networks, the data collection mode is changed to wireless. If the collected acceleration signal can be solved in the time domain, that is, the speed and displacement signals are obtained by accelerating the signal, this method will play a positive role in the health assessment of large-scale structure.

\subsection{Modal parameter identification method}

1) Frequency domain method

Modal parameter frequency domain identification method [9] is mainly using the measured frequency data or curve, according to the frequency response function modal expansion, to solve the system modal parameters. This method mainly includes early single-mode parameter identification method, multi-modal parameter identification method, nonlinear weighted least squares method and orthogonal polynomial fitting method. Single mode parameter identification method can meet the precision of smaller modal coupling system. Multi-modal parameter identification method is suitable for larger coupling systems. In general, we use the iterative method to complete the recognition of all modalities.

2) Time domain method

Modal parameter time domain identification method [10] also is called curve fitting method. The theoretical curve is fitted with the measured curve to minimize the error. Including the random decrement technique (RDT) method, the system identification modal parameter (ibrahim time domain -ITD) method, the least squares complex exponential (LSCE) method, the ARMA time series analysis method and the eigensystem realization algorithm (ERA) method.

Random decrement technique is a kind of vibration analysis method developed in the 1970s [11 13]. The ideas are as follows. We collect raw data which has several multiple random signals, remove the random response based on mean sample method, and identify the parameter modal according to the free response. It can rely on the excitation signal and only through the response signal to get the system modal parameters. The precondition is to make environment excitation signal as a random signal.

The basic idea of ITD identification is to collect the vibration signal of different measurement points for the vibration signals, and construct augmented matrix of free response in three different times [14]. The characteristic equations are established by 
the free response augmented matrix, so as to estimate the modal parameters of each order.

Least squares complex exponential method, also known as Prony polynomial method. The basic idea is to use the impulse response signal to identify the modal frequency and modal damping. Because this method is designed for a specific sampling point, so it belongs to the local recognition method, and can be identified by structure damage.

ARMA time series analysis method is one of the most commonly used methods in time domain analysis. The basic idea is to use the output-input signal to construct the difference equation and $\mathrm{Z}$ transform, so as to establish the equivalent relationship of the vibration equation and the ARMA model, the system function and the ARMA model, and then identify the modal parameters [15]. One of the key questions about this is how to effectively identify the parameters of the ARMA model.

The basic idea of ERA is to construct the Hankel matrix according to the collected system impulse response, and to construct the singularity decomposition of the matrix, so as to get the minimum implementation of the system. The system matrix is decomposed by the state matrix of the minimum implementation structure, and the modal parameters of the system are obtained.

3) Time-frequency method

The time-frequency method is mainly based on the wavelet transform analysis method. The time domain and frequency domain can be combined by wavelet transform. This method is often used for denoising or structural modal and damage identification based on wavelet packet energy spectrum.

\section{Time-domain integration error analysis of acceleration signal}

In practical engineering, the majority of product signals must not be found with the original function in the original function. With the increasing of digitization degree, the data acquisition mode is changed from wired to wireless, and Newton-Leibniz formula cannot be used directly, so we need to find a simple and effective time domain integration method.

The signal collected by the wireless sensor network is discrete signals, and we can express it when the sampling frequency is known. Time domain integration is the accumulation of area. There are many methods of time domain integration, including trapezoidal formula, middle rectangle formula, Simpson formula, Newton-Cortez formula, complex trapezoidal formula and so on.

We divide the interval $[a, b]$ into $n$ equal parts, $x_{k}=a+k h\left(h=\frac{b-a}{n}, k=0,1, \ldots, n\right)$, and apply trapezoidal formula in each subinterval 


$$
\begin{aligned}
& {\left[x_{k}, x_{k+1}\right](k=0,1, \ldots, n-1) \quad, \quad \int_{x_{k}}^{x_{k+1}} f(x) d x \approx \frac{h}{2}\left[f\left(x_{k}\right)+f\left(x_{k+1}\right)\right]} \\
& \text { then } I=\int_{a}^{b} f(x) d x=\sum_{k=1}^{n-1} \int_{x_{k}}^{x_{k+1}} f(x) d x=\frac{h}{2} \sum_{k=0}^{n-1}\left[f\left(x_{k}\right)+f\left(x_{k+1}\right)\right]+R_{n}(f), \text { and } R(f) \text { is truncation error. }
\end{aligned}
$$

Signals collected through a wireless sensor network are $\mathrm{a}(\mathrm{k}),(\mathrm{k}=1,2, \ldots, \mathrm{n})$.

Sampling frequency is $\mathrm{f}_{\mathrm{s}}$. One integration $\mathrm{a}(\mathrm{k})$ based on the above formula $\mathrm{v}(\mathrm{k}),(\mathrm{k}=1,2, \ldots, \mathrm{n}-1)$ is $\mathrm{v}(\mathrm{k})-\mathrm{v}(\mathrm{k}-1)=\frac{[\mathrm{a}(\mathrm{k})+\mathrm{a}(\mathrm{k}+1)]}{2 \mathrm{f}_{\mathrm{s}}}+\mathrm{R}_{\mathrm{k}}$, and then $\quad \mathrm{v}(\mathrm{k})=\mathrm{v}(\mathrm{k}-1)+\frac{[\mathrm{a}(\mathrm{k})+\mathrm{a}(\mathrm{k}+1)]}{2 \mathrm{f}_{\mathrm{s}}}+\mathrm{R}_{\mathrm{k}} \quad, \quad$ if $\quad \mathrm{v}(0)=0 \quad$, then $v(1)=\frac{[a(1)+a(2)]}{2 f_{s}}+R_{1}, v(2)=v(1)+\frac{[a(2)+a(3)]}{2 f_{s}}+R_{2}=\frac{a(1)+2 a(2)+a(3)}{2 f_{s}}+\left[R_{1}+R_{2}\right]$, $v(n-1)=v(n-2)+\frac{\lfloor a(n-1)+a(n)\rfloor}{2 f_{s}}+R_{n-1}$ $=\frac{a(1)+2 \sum_{i=2}^{n-1} a(i)+a(n)}{2 f_{s}}+\sum_{j=2}^{n-1} R_{j}$

$$
\mathrm{v}(\mathrm{n}-1) \approx \hat{v}(\mathrm{n}-1)=\frac{\mathrm{a}(1)+2 \sum_{\mathrm{i}=2}^{\mathrm{n}-1} \mathrm{a}(\mathrm{i})+\mathrm{a}(\mathrm{n})}{2 \mathrm{f}_{\mathrm{s}}} \text {. }
$$

In the one integration $\hat{v}(\mathrm{k})$, there will be a truncation error $\mathrm{R}_{\mathrm{k}}$, which means that high precision integral signal is relied one first order of truncation error precision, namely, the impact of accuracy of $\mathrm{h}$ and $\mathrm{n}$. At present, digital signal is the main source, and it involves the impact of integral accuracy of sampling frequency $f_{s}$ and sampling data length $\mathrm{n}$. We analyze the average error and mean square error in this paper.

If $\mathrm{a}(\mathrm{t})=\sin (0.6 \pi \mathrm{t})$, then $\mathrm{v}(\mathrm{t})=-0.6 \pi \cdot \cos (0.6 \pi \mathrm{t})$, from the time domain integration, we can obtain $\hat{v}(t)$. Mean error is $A M E=\frac{1}{n-1} \sum_{k=1}^{n-1}\left|f^{-1}(k)-f^{-1}(k)\right|$, mean square error is MSE $=\frac{1}{n-1} \sum_{k=1}^{n-1}\left[f^{-1}(k)-f^{-1}(k)\right]^{2}$.

1) when $n=1024$, the range of $f_{s}$ from $50 \mathrm{HZ}$ to $150 \mathrm{HZ}$, and the influence to AME is shown as Figure1. 


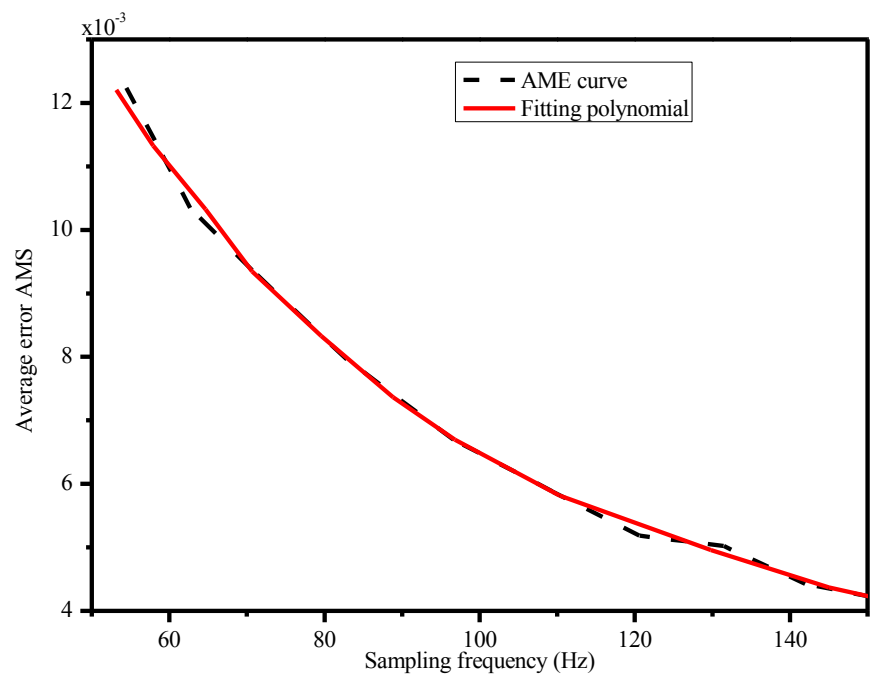

Fig. 1. Influence curve of frequency on AMS

The fitting polynomial function is $\mathrm{AME}=-7.2455 \times 10^{-9} \times\left(\mathrm{f}_{\mathrm{s}}\right)^{3}+2.9662 \times 10^{6} \times\left(\mathrm{f}_{\mathrm{s}}\right)^{2}-0.00044098 \times \mathrm{f}_{\mathrm{s}}+0.028034$, and the influence on MSE is shown as Figure 2.

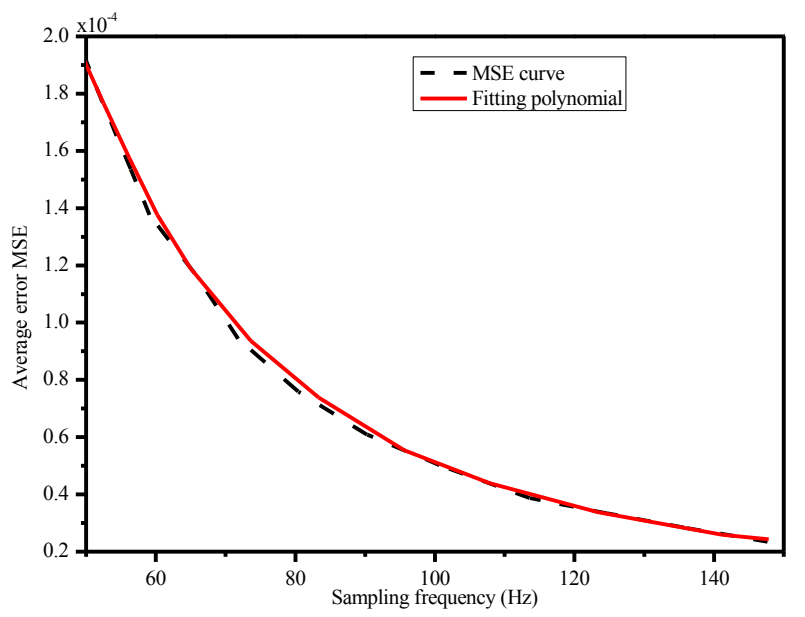

Fig. 2. Influence curve of frequency on MSE

\footnotetext{
The fitting polynomial function $\mathrm{MSE}=4.1078 \times 10^{-12} \times\left(\mathrm{f}_{\mathrm{s}}\right)^{4}-1.9641 \times 10^{-9} \times\left(\mathrm{f}_{\mathrm{s}}\right)^{3}+3.5597 \times 10^{-7} \times\left(\mathrm{f}_{\mathrm{s}}\right)^{2}$ $-2.9639 \times 10^{5} \times f_{s}+0.0010081$
} 
It can be seen from Fig. 1 and Fig. 2 that the sampling frequency $\mathrm{f}_{\mathrm{s}}$ has a great influence on the accuracy of time domain integration when the data length $\mathrm{n}$ is constant. When the sampling frequency $\mathrm{f}_{\mathrm{s}}$ is higher, the accuracy will be improved.

2) when $\mathrm{f}_{\mathrm{s}}=50, \mathrm{n}$ is 512, 1024, 2048, 4096 and 8192 respectively. The influence on AME and MSE is shown as Table 1.

Table 1. Influences of $\mathrm{n}$ on AME and MSE

\begin{tabular}{|c|c|c|c|c|c|}
\hline $\mathbf{n}$ & $\mathbf{5 1 2}$ & $\mathbf{1 0 2 4}$ & $\mathbf{2 0 4 8}$ & $\mathbf{4 0 9 6}$ & $\mathbf{8 1 9 2}$ \\
\hline $\begin{array}{c}\mathrm{AM} \\
\mathrm{E}\end{array}$ & 0.0126 & 0.0126 & 0.0128 & 0.0127 & 0.0127 \\
\hline $\mathrm{MSE}$ & $1.9438 \times 10^{-4}$ & $1.9567 \times 10^{-4}$ & $2.0055 \times 10^{-4}$ & $1.9948 \times 10^{-4}$ & $1.9983 \times 10^{-4}$ \\
\hline
\end{tabular}

As can be seen from Table 1, in the case of a fixed sampling frequency, the choice of data length $n$ has little effect on AME and MSE.
Therefore, the double

is $\mathrm{s}(\mathrm{k}),(\mathrm{k}=1,2, \ldots, \mathrm{n}-2) \cdot \mathrm{s}(\mathrm{k})=\mathrm{s}(\mathrm{k}-1)+\frac{\lfloor\mathrm{v}(\mathrm{k})+\mathrm{v}(\mathrm{k}+1)\rfloor}{2 \mathrm{f}_{\mathrm{s}}}+\mathrm{T}_{\mathrm{k}}$.

$$
\mathrm{s}(0)=0
$$

then

$$
\mathrm{s}(1)=\frac{[\mathrm{v}(1)+\mathrm{v}(2)]}{2 \mathrm{f}_{\mathrm{s}}}+\mathrm{T}_{1}
$$

$$
\begin{aligned}
& s(2)=s(1)+\frac{[v(2)+v(3)]}{2 f_{s}}+T_{2} \quad=\frac{2 a(1)+3 a(2)+a(3)}{\left(2 f_{s}\right)^{2}}+\left[\frac{2 R_{1}+R_{2}}{2 f_{s}}+T_{1}\right] \\
& =\frac{4 a(1)+7 a(2)+4 a(3)+a(4)}{\left(2 f_{s}\right)^{2}}+\left[\frac{4 R_{1}+3 R_{2}+R_{3}}{2 f_{s}}+T_{1}+T_{2}\right] \\
& s(n-2)=s(n-3)+\frac{[v(n-2)+v(n-1)]}{2 f_{s}}+T_{n-2}
\end{aligned},
$$

Similarly, when the sampling frequency $f_{s}$ is higher, the accuracy of time domain integration will be improved obviously. Data length $\mathrm{n}$ has little effect on precision.

From the above analysis, we can see that the higher sampling frequency $\mathrm{f}_{\mathrm{s}}$ well get higher precision of time domain integration. For wireless sensor networks, in order to 
ensure data transmission, sampling frequency is generally chosen as $f_{s}=100 \mathrm{HZ}$, which can guarantee the precision of time domain integration.

\section{$5 \quad$ Simulation results}

The network topology of this study is shown in Figure 3.

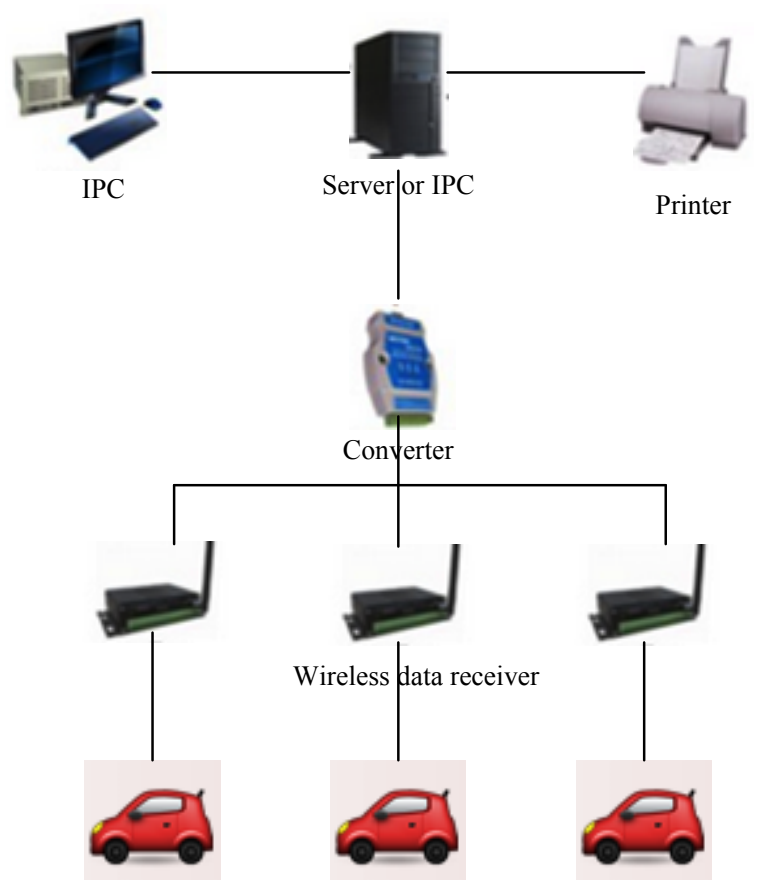

Fig. 3. Network topology

In the finite element modeling and simulation, we make the truck passes through the area, and we could get the acceleration, speed and displacement signal waveform of measuring point. This process can be regarded as a shock signal, and the vibration can be as the impulse response, and the initial value of it is zero, then the waveform is shown in Figure 4. 

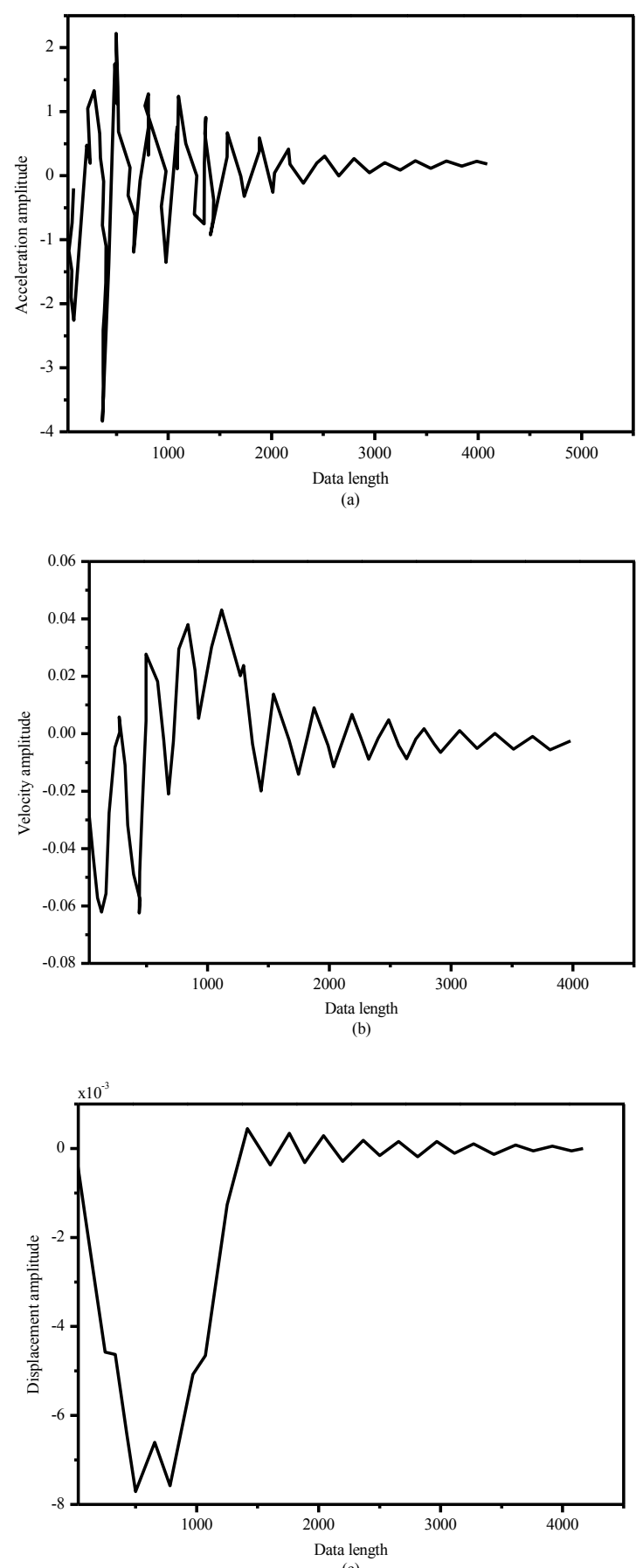

Fig. 4. Acceleration (a), speed (b) and displacement (c) signal waveform obtained by synchronous delay simulation 
By the above method, we can obtain the speed and displacement signal waveform based acceleration signal, which is shown in Figure 5. There is no trend term for the displacement signal waveform, so the initial value is zero.

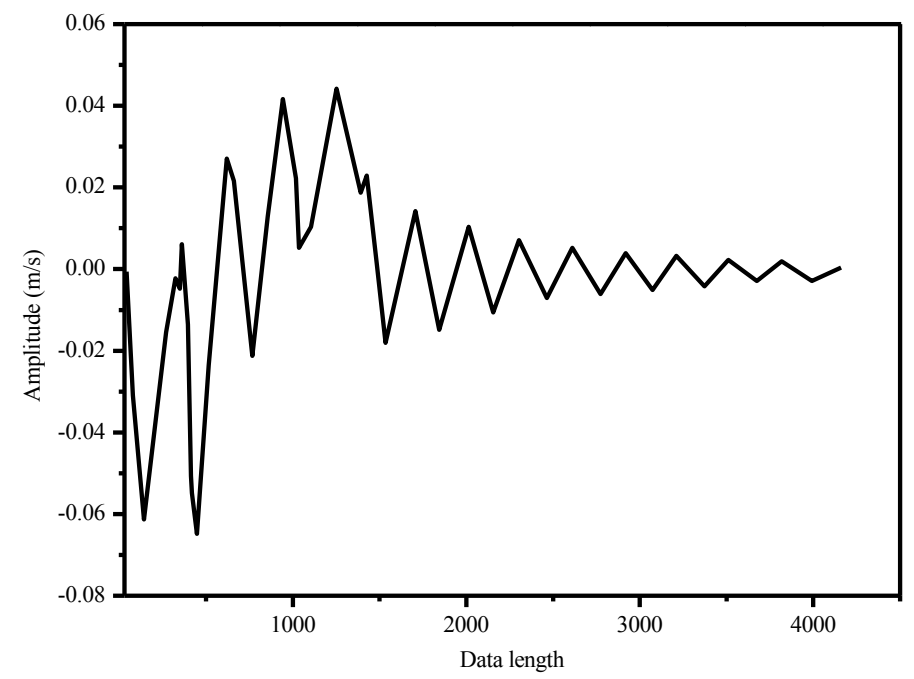

(a)

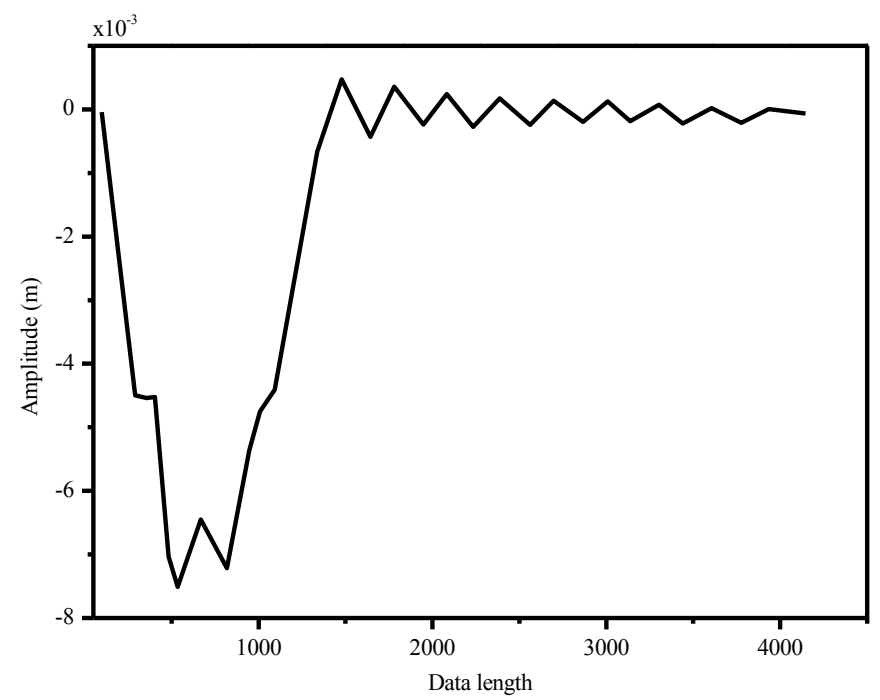

(b)

Fig.5. Speed (a), displacement (b) signal waveform based on acceleration signal 


\section{Conclusions}

The vibration signal (acceleration, velocity and displacement signals) is the basis for modal parameters, and it also is the basis of structural modal parameter identification. Through the displacement signal, we can obtain the operation and health status of structure. In the actual project, displacement sensor has many problems such as low precision, high price, installation difficulty and so on. Therefore, we can use the integral relation between acceleration, velocity, and the displacement signal to obtain the displacement signal. Based on the analysis of the relationship between the trend terms coefficients, this paper proposes a method of fast integration, so as to obtain speed, displacement signal of trend items elimination and get simulation instructions. In the case of further analysis of the fixed sampling frequency fs, we obtain the relationship between the data length $\mathrm{n}$ and the precision of the trend item, apply the conclusion to the acceleration signal processing acquired by the wireless sensor network and get corrected acceleration signal, velocity and displacement signal. Therefore, it is a necessary basis for follow-up large-scale structural modal identification.

\section{$7 \quad$ References}

[1] Assaf, Z. J., Petrov, D. A., \& Blundell, J. R. (2015). Obstruction of adaptation in diploids by recessive, strongly deleterious alleles. Proceedings of the National Academy of Sciences, 112(20), 2658-66. https://doi.org/10.1073/pnas.1424949112

[2] Burlayenko, V. N., Altenbach, H., \& Sadowski, T. (2015). An evaluation of displacementbased finite element models used for free vibration analysis of homogeneous and composite plates. Journal of Sound \& Vibration, 358, 152-175. https://doi.org/10.1016/j.jsv. 2015.08.010

[3] Elahi, A. S., Ghoranneviss, M., Elahi, A. S., \& Ghoranneviss, M. (2014). Discrete coils based measurement of plasma displacement in the ir-t1 tokamak. Journal of Nuclear \& Particle Physics, 4(1), 42-46.

[4] Han, F., Zhong, D. W., Mo, J. Y., \& Chen, H. (2014). Parameter identification for underground powerhouse of pumped-storage power station based on arma time-series model. Applied Mechanics \& Materials, 578-579, 1196-1199. https://doi.org/10.4028/www.scient ific.net/AMM.578-579.1196

[5] Hang, X. C., Jiang, L. W., Gu, M. H., Wu, S. Q., \& Fei, Q. G. (2015). Accuracy of modal damping identification using frequency domain decomposition method. Zhendong Gongcheng Xuebao/journal of Vibration Engineering, 28(4), 518-524.

[6] He, X. H., Hua, X. G., Chen, Z. Q., \& Huang, F. L. (2011). EMD-based random decrement technique for modal parameter identification of an existing railway bridge. Engineering Structures, 33(4), 1348-1356. https://doi.org/10.1016/j.engstruct.2011.01.012

[7] Kim, C. W., Kawatani, M., \& Hao, J. (2012). Modal parameter identification of short span bridges under a moving vehicle by means of multivariate AR model. Structure and Infrastructure Engineering, 8(5), 459-472. https://doi.org/10.1080/15732479.2010.539061

[8] Maekawa, A., Takahashi, T., Tsuji, T., \& Noda, M. (2015). Experimental validation of non-contacting measurement method using led-optical displacement sensors for vibration stress of small-bore piping.Measurement, 71, 1-10. https://doi.org/10.1016/j.mea surement.2015.04.008 
[9] Maekawa, A., Tsuji, T., Takahashi, T., \& Noda, M. (2014). A method using optical contactless displacement sensors to measure vibration stress of small-bore piping. Journal of Pressure Vessel Technology,136(1), 0112021-1120210.

[10] Meulenbeld, B. (2015). An accurate calculation method of vibration displacement based on vibration acceleration signal. Journal of Information \& Computational Science, 12(1), 41-49. https://doi.org/10.12733/jics20104848

[11] Squicciarini, G., Thompson, D. J., \& Corradi, R. (2014). The effect of different combinations of boundary conditions on the average radiation efficiency of rectangular plates. Journal of Sound \& Vibration, 333(17), 3931-3948. https://doi.org/10.1016/ j.jsv.2014.04.022

[12] Su, W. C., Liu, C. Y., \& Huang, C. S. (2014). Identification of Instantaneous Modal Parameter of Time - Varying Systems via a Wavelet - Based Approach and Its Application. Computer - Aided Civil and Infrastructure Engineering, 29(4), 279-298. https://doi.org/10.1111/mice.12037

[13] Wang, S., Huang, W., \& Zhu, Z. K. (2011). Transient modeling and parameter identification based on wavelet and correlation filtering for rotating machine fault diagnosis. Mechanical systems and signal processing, 25(4), 1299-1320. https://doi.org/10.1016/ j.ymssp.2010.10.013

[14] Yan, W. J., \& Ren, W. X. (2012). Operational modal parameter identification from power spectrum density transmissibility. Computer - Aided Civil and Infrastructure Engineering, 27(3), 202-217. https://doi.org/10.1111/j.1467-8667.2011.00735.x

[15] Zhang, J., Ouyang, H., Zhang, Y., \& Ye, J. (2015). Partial quadratic eigenvalue assignment in vibrating systems using acceleration and velocity feedback. Inverse Problems in Science and Engineering, 23(3), 479-497. https://doi.org/10.1080/17415977.2014.922076

\section{Authors}

Lian XUE is Professor at WuHan Technology And Business University, Wuhan 430065, China (vph9287506@126.com).

Cheng-song HU is Professor at WuHan Technology And Business University, Wuhan 430065, China (vfard9538364@126.com).

Article submitted 23 April 2017. Published as resubmitted by the authors 24 May 2017. 